patients were pregnant, their gender was unknown, or if there was a history of anaemia, iron deficiency, thalassaemia, haemochromatosis, inflammatory and infective conditions, or menorrhagia.

Results: 284,235 results were analysed. Ferritin and TSAT exhibited a significant decrease in the first 3 months of life, with concomitant increase in transferrin. Low ferritin flag rates peaked between 2 and 3 years, and again at 13 to 15 years, whereas high TSAT flagging was highest under 3 months for both genders, with a steady rise in boys over 15 years.

Conclusion: The appropriateness of age-related limits for iron studies in the paediatric population should be considered when interpreting results, especially in children under 3 months of age. References

1. Royal College of Pathologists of Australasia (RCPA). Iron Studies Standardised Reporting Protocol. Sydney: RCPA, 2013.

2. Heil W, Koberstein R, Zawta B. Reference Ranges for Adults and Children. 8th ed. Manheim, Germany: Roche Diagnostics, 2004.

\section{IMPACT OF THE 2020 VICTORIAN COVID LOCKDOWN MEASURES ON SERUM VITAMIN D LEVELS}

Michael B. Theophilos, Catherine Lynch, Jasmina Nguyen, Ken A. Sikaris

Melbourne Pathology, Collingwood, Vic, Australia

Introduction: In an attempt to curtail the spread of the SARSCoV-2 virus, the Victorian Government enacted a number of lockdown measures which confined many metropolitan residents to their homes for significant periods during 2020. We sought to examine whether there was any population-level effect on serum vitamin $\mathrm{D}$ levels resulting from extended home isolation during the Victorian 2020 winter.

Methods: A total of 3,839,232 serum Vitamin D measurements from the Melbourne Pathology database spanning the period January 2011 to May 2021 were extracted and analysed according to age, gender, and season.

Results: Analysis of results according to age, gender and season showed a reduction in overall vitamin D levels and an associated increase in the percentage of patients with vitamin D deficiency during the period of the second lockdown, but these results were comparable to the normal winter seasonal variation in previous years.

Discussion: Victoria entered its longest and most restrictive lockdown on 2nd August 2020, leading to speculation in the media that this could result in widespread deleterious reductions in Vitamin D levels. ${ }^{1}$ Our results show that the Victorian COVID lockdown measures had no significant effect on vitamin D levels when adjusted for age, gender and the normal Victorian seasonal variation. These results correlate with two retrospective studies from regions of Italy that experienced similar strict lockdown conditions. $^{2,3}$

\section{References}

1. 9 News. Coronavirus: Why lockdown could be making you vitamin D deficient. 10 Sep 2020. 9news.com.au/national/ coronavirus-why-lockdown-could-make-you-vitamin-d-deficient/47faaeba-84f5-4c4b-bd50-70e0c9486037

2. Lippi G, Ferrari A, Targher G. Is COVID-19 lockdown associated with vitamin D deficiency? Eur J Public Health 2021; 31: 278-9.
3. Ferrari D, Locatelli M, Faraldi M, et al. Changes in 25-(OH) vitamin D levels during the SARS-CoV-2 outbreak: lockdown-related effects and first-to-second wave difference-an observational study from Northern Italy. Biology 2021; 10: 237.

\section{CONVOLUTIONAL NEURAL NETWORKS ON POSTMODERN HISTOLOGY ORGAN RECOGNITION}

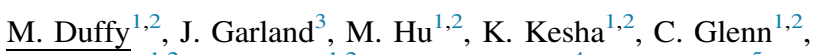
P. Morrow $^{1,2}$, S. Stables ${ }^{1,2}$, B. Ondruschka ${ }^{4}$, U. Da Broi ${ }^{5}$, R. Tse $\mathrm{T}^{1,2}$

${ }^{1}$ Faculty of Medical and Health Sciences, University of Auckland, Auckland, New Zealand; ${ }^{2}$ Department of Forensic Pathology, LabPLUS, Auckland City Hospital, Auckland, New Zealand; ${ }^{3}$ Forensic and Analytical Science Service, NSW Health Pathology, NSW, Australia; ${ }^{4}$ Institute of Legal Medicine, University Medical Center Hamburg-Eppendorf, Hamburg, Germany; and ${ }^{5}$ Department of Medicine, Section of Forensic Medicine, University of Udine, Udine, Italy

Convolutional neural network $(\mathrm{CNN})$ has advanced in recent years and translated from research into medical practice. Research on CNNs in forensic/post mortem pathology is almost exclusive to post mortem computed tomography, despite the wealth of research into CNNs in surgical/ anatomical histopathology. This study was carried out to investigate whether CNNs are able to recognise different organs on histology slides. This study compared four CNNs commonly used in surgical/anatomical histopathology to identify microscopic images of brain, heart, kidney, lung and liver. One of the CNNs used (InceptionResNet v2) was able to show a $>95 \%$ accuracy in classifying the organs. The result of this study is promising and demonstrates that CNN technology has potential applications as a screening and probably a computer assisted diagnostics tool in forensic/post mortem histopathology.

\section{AN INVESTIGATION OF CHANGING FORENSIC PATHOLOGY WORKLOADS IN THE CORONIAL JURISDICTIONS OF AUSTRALIA}

Lana Matteucci $^{1}$, Charles Naylor ${ }^{2,3}$, Neil Langlois ${ }^{1,4}$

${ }^{1}$ University of Adelaide, SA, Australia; ${ }^{2}$ Forensic and Scientific Services Queensland Health, Qld, Australia; ${ }^{3}$ University of Queensland, Qld, Australia; and ${ }^{4}$ Forensic Science SA, SA, Australia

The workload of forensic pathologists involves performing autopsies for the coroner. Over the period 2006-2016 it was expected to see a change in autopsy workload of forensic pathologists in Australia due to the increasing number of deaths per year. This study investigated if trends in Australian coronial autopsy workload are related to changes in Coroners Acts. Analysis was performed using data from the National Coronial Information System (NCIS), which was compared with the death data from the Australian Bureau of Statistics (ABS) and Coroners Acts between 2006-2016. In every jurisdiction, the number of deaths per year increased during the study period, but not all jurisdictions observed an increase number of autopsies per year. In three of the eight coronial jurisdictions of 